\title{
Perfil sociodemográfico e obstétrico dos óbitos fetais de gestantes residentes em um município do estado do Maranhão
}

\author{
Sociodemographic and obstetric profile of fetal deaths of pregnant women living in a \\ municipality in the state of Maranhão
Perfil sociodemográfico y obstétrico de muertes fetales de mujeres embarazadas que viven en un municipio en el estado de Maranhão

Letícia Samara Ribeiro da Silva ${ }^{1 *}$, Patrícia Samara Ribeiro da Silva ${ }^{1}$, Maria Cleilda Araujo Santos ${ }^{1}$, Andressa Arraes Silva ${ }^{1}$, Larissa Silva Oliveira ${ }^{1}$, Maria Beatriz Pereira da Silva ${ }^{1}$, Ana Cláudia de

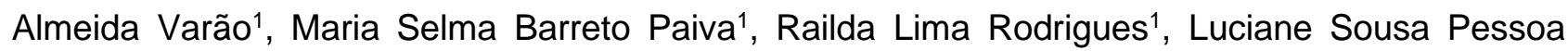
Cardoso ${ }^{1}$.

\section{RESUMO}

Objetivo: Analisar o perfil sociodemográfico e obstétrico dos óbitos fetais de gestantes residentes em um município do Maranhão, entre 2014 e 2018. Métodos: Estudo descritivo, retrospectivo, com abordagem quantitativa, realizado com base em dados do Sistema de Informação sobre Mortalidade. Foram consideradas variáveis relacionadas à mãe, ao feto, ao parto e a causa do óbito conforme a CID-10. Os dados foram analisados e tabulados utilizando-se o software Microsoft Excel. Não houve necessidade de submissão ao Comitê de Ética em Pesquisa devido à natureza pública dos dados. Resultados: Os resultados evidenciaram 114 óbitos fetais, na maioria em gestantes com idade entre 20 e 29 anos, 8 a 11 anos de estudo e gestação única. Houve predominância de óbitos em fetos do sexo masculino, com idade gestacional entre 37 e 41 semanas e peso ao nascer de 1500 a 2499 gramas. Observou-se maior frequência em partos vaginais, no anteparto e ocorridos em hospitais. As principais causas foram Hipóxia intrauterina, Complicações da placenta, cordão umbilical e membranas e outras afecções originadas no período perinatal. Conclusão: Espera-se com este estudo fornecer informações úteis que subsidiem a formulação e a implementação de ações que visem a prevenção e redução dos óbitos fetais no município.

Palavras-chave: Registros de mortalidade, Morte fetal, Causas de morte.

\begin{abstract}
Objective: To analyze the sociodemographic and obstetric profile of fetal deaths of pregnant women living in a municipality in Maranhão, between 2014 and 2018. Methods: Descriptive, retrospective study with quantitative approach, based on data from the Mortality Information System. Variables related to the mother, fetus, childbirth and cause of death according to ICD-10 were considered. The data were analyzed and tabulated using Microsoft Excel software. There was no need to submit to the Research Ethics Committee due to the public nature of the data. Results: The results showed 114 fetal deaths, mostly in pregnant women aged between 20 and 29 years, 8 to 11 years of study and single pregnancy. There was a predominance of deaths in male fetuses, with gestational age between 37 and 41 weeks and birth weight of 1500 to 2499 grams. A higher frequency was observed in vaginal deliveries, in the antepartum and in hospitals. The main causes were intrauterine hypoxia, complications of the placenta, umbilical cord and membranes and other conditions
\end{abstract}

${ }^{1}$ Universidade Estadual do Maranhão (UEMA), Bacabal - MA. *E-mail: leticiasamaras2@outlook.com

SUBMETIDO EM: 2/2020

ACEITO EM: 3/2020

PUBLICADO EM: 4/2020

REAS/EJCH | Vol.Sup.n.45 | e3113 | DOI: https://doi.org/10.25248/reas.e3113.2020 Página 1 de 9 
originating in the perinatal period. Conclusion: This study is expected to provide useful information to support the formulation and implementation of actions aimed at preventing and reducing fetal deaths in the municipality.

Key words: Mortality records, Fetal death, Causes of death.

\section{RESUMEN}

Objetivo: Analizar el perfil sociodemográfico y obstétrico de las muertes fetales de mujeres embarazadas que viven en un municipio de Maranhão, entre 2014 y 2018. Métodos: Estudio descriptivo, retrospectivo, con enfoque cuantitativo, realizado a partir de datos del Sistema de Información de Mortalidad. Las variables relacionadas con la madre, el feto, el parto y la causa de la muerte de acuerdo con ICD-10 se consideraron. Los datos fueron analizados y tabulados utilizando el software Microsoft Excel. No hubo necesidad de enviarlo al Comité de Ética de Investigación debido a la naturaleza pública de los datos. Resultados: Los resultados mostraron 114 muertes fetales, principalmente en mujeres embarazadas de entre 20 y 29 años, de 8 a 11 años de estudio y embarazo único. Hubo un predominio de muertes en fetos masculinos, con edad gestacional entre 37 y 41 semanas y peso al nacer de 1500 a 2499 gramos. Se observó una mayor frecuencia en partos vaginales, en el anteparto y en hospitales. Las causas principales fueron hipoxia intrauterina, complicaciones de la placenta, el cordón umbilical y las membranas y otras afecciones originadas en el período perinatal. Conclusión: Se espera que este estudio brinde información útil para apoyar la formulación e implementación de acciones destinadas a prevenir y reducir las muertes fetales en el municipio.

Palabras clave: Registros de mortalidad, Muerte fetal, Causas de muerte.

\section{INTRODUÇÃO}

O óbito fetal constitui-se como um significativo problema de saúde pública no Brasil. É definido, de acordo com o Ministério da Saúde, como a morte do produto da gestação antes da expulsão ou completa extração do organismo da mãe, independentemente do tempo da gravidez. Determina o óbito o fato de o feto não respirar nem dar qualquer outro sinal de vida depois de ocorrer a separação (BRASIL, 2009).

Conforme Barros OS, et al. (2019), em 2016, foram registrados 1,7 milhões de óbitos fetais no mundo, representando uma diminuição de 65,3\% desde 1970. No Brasil, de 2000 a 2015 também se observou uma redução de quase $30 \%$ nesses óbitos, o que pode ser explicado devido ao aumento da cobertura de atenção ao pré-natal. No entanto, o número ainda é alto quando se leva em consideração outros países em desenvolvimento, como Chile e Argentina (SCHRADER G, 2017).

Na literatura brasileira, os estudos que abordam o óbito fetal, suas causas básicas e fatores relacionados ainda são escassos quando comparados àqueles que tratam da mortalidade infantil como um todo (GIRALDI LM, et al., 2019). Somado a isso, segundo Schrader G (2017), apesar de o monitoramento das taxas de mortalidade fetal e infantil ser de forma conjunta um dos eixos estratégicos da Política Nacional de Atenção Integral à Saúde da Criança, os programas e políticas de saúde ainda focalizam em sua maioria o óbito infantil.

Mesmo tendo sido historicamente negligenciados pelos serviços de saúde, a despeito de sua grande importância, estudos apontam algumas condições que favorecem a ocorrência do óbito fetal, dentre elas: deficiente qualidade da assistência prestada pelos profissionais de saúde tanto durante o pré-natal quanto no momento do parto; características maternas, como idade, escolaridade, doenças prévias e perdas fetais anteriores; características da gravidez, como a gemelaridade, e do feto, como idade gestacional e peso (HOLANDA AAS, 2013; SCHRADER G, 2017).

Tendo em vista que a mortalidade fetal reflete a qualidade e as condições de acesso a cuidados de saúde disponibilizados desde a Atenção Primária às gestantes, bem como a qualidade da assistência intraparto, é fundamental conhecer e entender a epidemiologia da morte fetal a fim de promover ações voltadas à melhoria da saúde materno-infantil, subsidiando a adoção de medidas preventivas em relação aos óbitos fetais, os 
quais são considerados, em sua maioria, como eventos potencialmente evitáveis por ações básicas realizadas nos serviços de saúde (MENEZZI AMED, et al., 2016). Como afirma Schrader G (2017), a caracterização dos fatores associados à ocorrência do óbito fetal, aliado ao conhecimento do seu perfil em determinado local, proporcionam também a criação ou readequação de protocolos que objetivam o seu controle e que sejam adaptados ao contexto local.

Nesse contexto, considerando a necessidade de mais estudos que abordem essa temática e que o óbito fetal, apesar de ser o menos valorizado entre os itens da mortalidade em geral, se constitui como um relevante problema de saúde materno-infantil, o presente estudo tem como objetivo analisar o perfil sociodemográfico e obstétrico dos óbitos fetais de gestantes residentes em um município do Maranhão, no período de 2014 a 2018.

\section{MÉTODOS}

Trata-se de um estudo de caráter descritivo, retrospectivo, com abordagem quantitativa, realizado com base em dados secundários obtidos através do Sistema de Informação sobre Mortalidade (SIM), por meio do acesso ao Departamento de Informática do Sistema Único de Saúde (DATASUS). A coleta de dados ocorreu no mês de janeiro de 2020. Foram incluídos todos os óbitos fetais de gestantes residentes em um município do Maranhão, notificados no período de 2014 a 2018. Foram excluídos os dados não referentes ao período em estudo. Tendo em vista a dimensão do óbito fetal como problema a ser enfrentado e as adversidades a ele associadas, este estudo teve como pergunta norteadora: Qual o perfil sociodemográfico e obstétrico dos óbitos fetais de gestantes residentes em um município do estado do Maranhão, no período de 2014 a 2018 ?

Para a análise desse perfil, foram consideradas as seguintes variáveis: relacionadas à mãe: idade (10 a 19 anos, 20 a 29 anos, 30 a 39 anos, 40 a 49 anos), escolaridade (nenhuma, 1 a 3 anos, 4 a 7 anos, 8 a 11 anos, 12 anos ou mais), tipo de gravidez (única, dupla); relacionadas ao feto: sexo (masculino, feminino), raça/cor, duração da gestação (menos de 22 semanas, 22 a 27 semanas, 28 a 31 semanas, 32 a 36 semanas, 37 a 41 semanas), peso ao nascer (menos de 500g, 500 a 999g, 1000 a 1499 g, 1500 a 2499 g, 2500 a 2999 g, 3000 a 3999 g, 4000g e mais); relacionadas ao parto: tipo de parto (vaginal, cesáreo), óbito em relação ao parto (antes do parto, durante o parto), local de ocorrência (hospital, outro estabelecimento de saúde, domicílio, via pública) e causa do óbito, esta definida utilizando-se a Classificação Estatística Internacional de Doenças e Problemas Relacionados à Saúde (CID-10).

Os dados foram analisados e tabulados utilizando-se o software Microsoft Excel, versão 2010, sendo apresentados em tabelas em frequência absoluta e percentual. Não houve necessidade de submissão ao Comitê de Ética em Pesquisa devido à natureza pública dos dados.

\section{RESULTADOS}

No período de 2014 a 2018, foram notificados 114 óbitos fetais de gestantes residentes em um município do Maranhão, sendo que em 2015 houve a maior frequência desses óbitos, somando um total de 36 (31\%). Em contrapartida, a menor frequência ocorreu no ano de 2016 com 17 óbitos (15\%) (Tabela 1).

Tabela 1 - Número de óbitos fetais segundo ano de ocorrência. 2014-2018.

\begin{tabular}{ccc}
\hline Ano do Óbito & $\mathbf{n}$ & $\%$ \\
\hline 2014 & 24 & 21 \\
2015 & 36 & 31 \\
2016 & 17 & 15 \\
2017 & 18 & 16 \\
2018 & 19 & 17 \\
\hline Total & 114 & 100 \\
\hline
\end{tabular}

Fonte: Silva LSR, et al., 2020; SIM, 2020.

De acordo com os dados maternos encontrados, verificou-se a predominância da idade da mãe entre 20 e 29 anos, totalizando 61 óbitos fetais (53\%); média escolaridade dessas gestantes, com maior frequência de 
8 a 11 anos de estudo (55\%; n=63); e quanto ao tipo de gravidez, dos 114 óbitos fetais, 107 (94\%) ocorreram em gestações únicas (Tabela 2).

Tabela 2 - Óbitos fetais segundo as variáveis relacionadas à mãe. 2014-2018.

\begin{tabular}{ccc}
\hline Variável & $\mathbf{n}$ & $\%$ \\
\hline Idade da mãe & & \\
\hline 10 a 19 anos & 25 & 22 \\
20 a 29 anos & 61 & 53 \\
30 a 39 anos & 20 & 18 \\
40 a 49 anos & 2 & 2 \\
Ignorada & 6 & 5 \\
\hline Escolaridade da mãe (anos de estudo) & & \\
\hline Nenhuma & 4 & 4 \\
1 a 3 anos & 9 & 8 \\
4 a 7 anos & 26 & 23 \\
8 a 11 anos & 63 & 55 \\
12 anos ou mais & 5 & 4 \\
Ignorado & 7 & 6 \\
\hline Tipo de gravidez & & \\
Única & 107 & 94 \\
Dupla & 6 & 5 \\
Ignorada & 1 & 1 \\
Total & 114 & 100 \\
\hline
\end{tabular}

Fonte: Silva LSR, et al., 2020. SIM, 2020.

No que se refere às variáveis relacionadas ao feto, o maior número de óbitos ocorreu no sexo masculino, o que correspondeu a 63 óbitos fetais (55\%). Já com relação à variável raça/cor, não foi possível a realização de sua análise estatística por ser um dado ignorado em 100\% dos óbitos fetais. Com relação à duração da gestação, a maioria dos óbitos fetais ocorreu entre a $37^{a}$ e a $41^{\text {a }}$ semana $(n=30)$, correspondendo a $26,3 \%$. Os dados também apontam baixo peso ao nascer (<2500 gramas), com predominância de fetos com peso de 1500 a 2499 gramas (25\%; n=29) (Tabela 3).

Tabela 3 - Óbitos fetais segundo as variáveis relacionadas ao feto. 2014-2018.

\begin{tabular}{|c|c|c|}
\hline Variável & $\mathbf{n}$ & $\%$ \\
\hline \multicolumn{3}{|l|}{ Sexo } \\
\hline Masculino & 63 & 55 \\
\hline Feminino & 51 & 45 \\
\hline \multicolumn{3}{|l|}{ Raça/cor } \\
\hline Ignorado & 114 & 100 \\
\hline \multicolumn{3}{|l|}{ Duração da gestação } \\
\hline Menos de 22 semanas & 11 & 9,6 \\
\hline 22 a 27 semanas & 21 & 18,4 \\
\hline 28 a 31 semanas & 17 & 15 \\
\hline 32 a 36 semanas & 22 & 19,3 \\
\hline 37 a 41 semanas & 30 & 26,3 \\
\hline Ignorado & 13 & 11,4 \\
\hline \multicolumn{3}{|l|}{ Peso ao nascer } \\
\hline Menos de $500 \mathrm{~g}$ & 2 & 2 \\
\hline 500 a $999 g$ & 23 & 20 \\
\hline 1000 a $1499 \mathrm{~g}$ & 23 & 20 \\
\hline 1500 a $2499 \mathrm{~g}$ & 29 & 25 \\
\hline 2500 a $2999 \mathrm{~g}$ & 12 & 11 \\
\hline 3000 a $3999 \mathrm{~g}$ & 18 & 16 \\
\hline $4000 \mathrm{~g}$ e mais & 1 & 1 \\
\hline Ignorado & 6 & 5 \\
\hline Total & 114 & 100 \\
\hline
\end{tabular}

Fonte: Silva LSR, et al., 2020. SIM, 2020. 
Quando se analisou a proporção de óbitos fetais segundo as variáveis inerentes ao parto, observou-se um maior percentual em partos via vaginal $(64 \% ; n=73)$. Demonstra-se, também, uma alta frequência de óbitos ocorridos antes do parto, num total de 110 (96\%) dos 114 óbitos fetais analisados. Já quanto ao local de ocorrência, quase totalidade dos óbitos fetais $(92,1 \% ; n=105)$ ocorreram em hospitais (Tabela 4).

Tabela 4 - Óbitos fetais segundo as variáveis relacionadas ao parto. 2014-2018.

\begin{tabular}{ccc}
\hline Variável & $\mathbf{n}$ & $\%$ \\
\hline Tipo de parto & & \\
\hline Vaginal & 73 & 64 \\
Cesáreo & 40 & 35 \\
Ignorado & 1 & 1 \\
\hline Óbito em relação ao parto & & \\
\hline Antes do parto & 110 & 96 \\
Durante o parto & 1 & 1 \\
Após o parto & 1 & 1 \\
Ignorado & 2 & 2 \\
\hline Local de ocorrência & & \\
\hline Hospital & 105 & 92,1 \\
Outro estabelecimento de saúde & 4 & 2,6 \\
Domicílio & 3 & 0,9 \\
Via pública & 1 & 0,9 \\
Ignorado & 1 & 100 \\
\hline Total & 114 &
\end{tabular}

Fonte: Silva LSR, et al., 2020. SIM, 2020.

No que concerne à causa do óbito, no período em estudo, 112 óbitos fetais (98\%) tiveram sua causa básica relacionada ao capítulo XVI da CID-10 (Algumas afecções originadas no período perinatal) e apenas dois óbitos (2\%) incluíam o capitulo XVII (Malformações congênitas, deformidades e anomalias cromossômicas) como causa do óbito.

Tabela 5 - Causas dos óbitos fetais segundo categoria da CID-10. 2014-2018.

\begin{tabular}{lcc}
\hline \multicolumn{1}{c}{ Causa do óbito } & $\mathbf{n}$ & $\%$ \\
\hline $\begin{array}{l}\text { P00 Feto e recém-nascido afetados por afecções maternas, não } \\
\text { obrigatoriamente relacionadas com a gravidez atual }\end{array}$ & 7 & 6 \\
$\begin{array}{l}\text { P01 Feto e recém-nascido afetados por complicações maternas } \\
\text { da gravidez }\end{array}$ & 2 & 2 \\
$\begin{array}{l}\text { P02 Feto e recém-nascido afetados por complicações da } \\
\text { placenta, do cordão umbilical e das membranas }\end{array}$ & 19 & 17 \\
P07 Transtornos relacionados com a gestação de curta duração e & 1 & 1 \\
peso baixo ao nascer não classificados em outra parte & 38 & 33 \\
P20 Hipóxia intrauterina & 1 & 1 \\
P70 Transtornos transitórios do metabolismo dos carboidratos & 1 & \\
específicos do feto e do recém-nascido & 1 & 1 \\
P83 Outras afecções comprometendo o tegumento específicas do & 1 & \\
feto e do recém-nascido & 29 & 25 \\
P95 Morte fetal de causa não especificada & 14 & 12 \\
P96 Outras afecções originadas no período perinatal & 2 & 2 \\
Q00 Anencefalia e malformações similares & 114 & 100 \\
\hline Total &
\end{tabular}

No que diz respeito ao capítulo XVI da CID-10, as quatro categorias com maior número de óbitos fetais foram: Hipóxia intrauterina com 38 óbitos (33\%), seguido de 29 mortes fetais (25\%) de causa não especificada, 19 fetos (17\%) afetados por Complicações da placenta, do cordão umbilical e das membranas, 
e $14(12 \%)$ provocados por Outras afecções originadas no período perinatal. Quanto ao capítulo XVII, foram registrados dois óbitos (2\%) inclusos na categoria Anencefalia e malformações similares. As demais causas estão descritas a seguir (Tabela 5).

\section{DISCUSSÃO}

O óbito fetal é considerado um importante indicador de saúde, sendo o mais apropriado para analisar a qualidade da assistência obstétrica prestada tanto no período gestacional quanto no parto (LIMA KJ, et al., 2017). Conforme Mazotti BR, et al. (2016), uma vez que fatores socioeconômicos, demográficos, patológicos e assistenciais aliados a características maternas, do feto e do parto contribuem para o entendimento do óbito fetal, e pela importância epidemiológica do tema, tem-se como objetivo analisar o perfil sociodemográfico e obstétrico dos óbitos fetais de gestantes residentes em um município do Maranhão, no período de 2014 a 2018.

Pode-se constatar neste estudo, quanto à idade materna, um elevado percentual de óbitos fetais de gestantes com idade inferior a 30 anos (75\%), estando mais da metade (53\%) entre 20 e 29 anos. A associação entre essa variável com o evento da morte fetal apresenta-se de forma divergente da literatura, pois em mulheres de 20 a 35 anos de idade a gravidez é considerada como de baixo risco (FILHA FSSC, et al., 2019). O número maior de nascimentos para as gestantes incluídas nessa faixa etária e a preocupação das mulheres em não engravidar em idades consideradas perigosas, principalmente após os 35 anos, são possíveis explicações para esse fato (BRITO MAMM, et al., 2019).

Com relação à escolaridade das mães, em oposição a outros estudos que apontam a falta ou baixa escolaridade como fator de risco para o óbito fetal, demonstrou-se maior frequência entre gestantes com 8 a 11 anos de estudo (55\%). Esse resultado corrobora com o estudo realizado por Barros PS, et al. (2019), o qual traçou uma série histórica da mortalidade fetal no Brasil e regiões entre 1996 e 2015, identificando seu comportamento e tendência. Nele, evidenciou-se que ocorreu uma diminuição da proporção de óbitos fetais para mães com até sete anos de escolaridade, mas aumentou em mães com mais de oito anos de estudo.

No que se refere ao tipo de gestação, constatou-se um maior número de óbitos fetais ocorridos em gestações únicas (94\%). Verifica-se, neste estudo, que não houve associação significativa desse desfecho obstétrico com a gestação gemelar (presença de dois ou mais fetos na cavidade uterina), apesar de, segundo - Ministério da Saúde, estar associada a um maior risco de óbito fetal (BRASIL, 2012). Dados semelhantes são evidenciados nos estudos de Holanda AAS (2013), Schrader G (2017) e Brito MAMM, et al. (2019), o que pode ser explicado devido a maior quantidade do tipo de gravidez única em relação à múltipla.

Outra variável analisada foi com relação ao sexo do natimorto. A maior parte dos óbitos fetais ocorreu no sexo masculino (55\%). Esse resultado vai de encontro à Teoria da vulnerabilidade biológica inata masculina. Chiavegatto-Filho ADP e Laurenti R (2012), em seu estudo intitulado "O sexo masculino vulnerável: razão de masculinidade entre os óbitos fetais brasileiros", afirmam que situações de estresse levariam a um aumento da mortalidade fetal masculina devido à maior vulnerabilidade dos fetos desse sexo aos corticosteroides liberados em situações extremas. Schrader G (2017) também enfatiza essa teoria ao afirmar que outros estudos também já associaram essa maior ocorrência de óbitos fetais no sexo masculino ao fato de que a maturação pulmonar ocorre de forma mais tardia do que em fetos do sexo feminino, o que, consequentemente, aumenta as chances de desenvolverem problemas respiratórios que levariam ao óbito.

Quando se analisou a duração da gestação, a maioria dos óbitos fetais ocorreu entre 37 e 41 semanas. 0 mesmo resultado foi encontrado no estudo de Lima KJ, et al. (2017), no qual mostram que a maior parte das mortes fetais ocorreu em gestações tardias. $O$ dado se mostra relevante uma vez que contraria muitas das evidências científicas que associam a menor duração da gestação com o risco de óbito fetal, como os estudos de Holanda AAS (2013) e Barros PS, et al. (2019), os quais apontam uma maior frequência de natimortos com idade gestacional entre 22 e 36 semanas, considerados fetos pré-termos ou pré-termos extremos. Nesse contexto, destaca-se que a ocorrência de mortes fetais no final da gestação demostra a relação entre esses óbitos e a qualidade do atendimento às gestantes, o que leva a supor que a assistência pré-natal não está 
sendo satisfatória, visto que o feto entre 37 e 41 semanas é considerado a termo e tem grande potencial de sobrevivência (BRASIL, 2009; LIMA KJ, et al., 2017).

No que diz respeito ao peso ao nascer, verificou-se a predominância do peso fetal entre 1500 a 2499 gramas. Tendo em vista a classificação do Ministério da Saúde (2009) que considera: recém-nascido de extremo baixo peso - peso ao nascer $<1.000$ gramas; recém-nascido de muito baixo peso - peso ao nascer $<1.500$ gramas; recém-nascido de baixo peso - peso ao nascer $<2500$ gramas; e peso adequado $>2500$ gramas, neste estudo $67 \%$ dos óbitos ocorreram em fetos com peso ao nascer <2500 gramas, denotando maior frequência de baixo peso entre os casos estudados de morte fetal. Esse resultado assemelha-se à maioria dos estudos disponíveis, como o de Oliveira EFV, et al. (2010), no qual analisam que o risco de óbito fetal é aumentado em 5,72 vezes quando o peso do feto é inferior a 2500 gramas.

Também é importante destacar que, embora tenha ocorrido um número maior de óbitos fetais com baixo peso, houve ainda 31 óbitos de fetos com peso acima de 2499 gramas, considerados dentro do peso adequado e que, teoricamente, estariam aptos ao nascimento. Essa informação ressalta uma falha na atenção à saúde das gestantes, uma vez que a morte fetal está ocorrendo em condições que poderiam ser evitadas (SILVA RMM, et al., 2016).

Levando em consideração o tipo de parto, fica evidente o alto percentual de casos de óbitos fetais relacionados ao parto vaginal (64\%), o mesmo resultado encontrado nos estudos de Schrader G (2017), Barros PS, et al. (2019) e Giraldi LM, et al. (2019). Conforme Schrader G (2017), esse resultado fortalece algumas evidências científicas de que o parto cesáreo abrevia o tempo de duração do trabalho de parto, evitando que o óbito fetal ocorra. Porém, como afirmam Barros PS et al. (2019), os partos cesáreos devem ser realizados em circunstâncias bem definidas, já que, comparado ao parto vaginal, estão associados a um aumento da morbimortalidade materna e neonatal em curto e longo prazo, além de cesarianas anteriores favorecerem de forma significativa o risco para o óbito fetal em gestações futuras, podendo aumentar esses óbitos em até 23\% (SILVA RMM, et al., 2016).

Quanto a variável óbito em relação ao parto, observou-se que os óbitos fetais ocorreram predominantemente antes do parto (96\%), assim como apontam os estudos realizados por Lima KJ, et al. (2017), Silva MMB, et al. (2017) e Brito MAMM, et al. (2019). Para a análise desses óbitos, têm sido utilizadas algumas classificações de evitabilidade de acordo com a possibilidade de prevenção, dentre as quais 0 Ministério da Saúde recomenda a Classificação de Wigglesworth (BRASIL, 2009).

Como afirma Schrader G (2017), de acordo com a Classificação de Evitabilidade de Wigglesworth Expandida por grupo de causas, os óbitos fetais ocorridos no período anteparto estão geralmente relacionados a falhas na atenção prestada durante o pré-natal e com condições adversas inerentes à mãe. Já os óbitos ocorridos no período intraparto estariam relacionados a falhas no manejo obstétrico, como traumas no momento do parto e asfixia (LIMA KJ, et al., 2017). Pode-se levar a crer, dessa forma, na existência de deficiências na assistência fornecida pelos profissionais de saúde às gestantes. Destaca-se, um óbito (1\%) informado como de ocorrência depois do parto, o que constitui uma incoerência com a definição de óbito fetal ou mesmo um erro no preenchimento da Declaração de Óbito.

Já em relação ao local de ocorrência dos óbitos fetais, foi evidenciado neste estudo que 95,6\% ocorreram em estabelecimentos de saúde, com predominância de $92,1 \%$ ocorridos em hospitais. Esse resultado confirma a preferência do parto hospitalar no Brasil, em que, de acordo com o Ministério da Saúde, $98 \%$ dos partos são realizados em ambiente hospitalar (BRASIL, 2009).

No que concerne à causa do óbito fetal, o presente estudo aponta como principais causas a Hipóxia intrauterina (33\%), Complicações da placenta, do cordão umbilical e das membranas (17\%) e outras afecções originadas no período perinatal (12\%). A maior frequência de óbitos registrados tendo como causa básica a hipóxia intrauterina, assim como demonstrado no estudo de Schrader G (2017), não contribui de forma significativa para a compreensão do óbito fetal, uma vez que, em algumas situações, se torna difícil definir a cadeia de eventos que levou ao óbito (BRASIL, 2009; BARBEIRO FMS, et al., 2015). Somado a isso, é importante destacar o elevado percentual de mortes fetais de causa não especificada (25\%), o que evidencia 
a importância da investigação do óbito para identificar sua etiologia e facilitar a adoção de medidas preventivas, como preconizado na Portaria $\mathrm{n}^{\circ}$ 72, de 11 de janeiro de 2010, que estabelece a obrigatoriedade da vigilância do óbito infantil e fetal nos serviços de saúde, sejam públicos ou privados (BRASIL, 2010; ASSIS HM, et al., 2014).

Por meio deste estudo, reforça-se a importância de uma assistência adequada desde o pré-natal, com os cuidados disponibilizados na Atenção Primária, até a assistência hospitalar ao parto, além do reconhecimento de situações de risco que possam desencadear o óbito fetal (SILVA VMC, et al., 2019). É imprescindível que os programas e políticas públicas estejam direcionados também à prevenção dos óbitos fetais, com investimentos específicos para a sua redução (BARROS PS, et al., 2019). Ademais, os profissionais de saúde precisam incorporar em sua rotina de trabalho a análise da ocorrência desses óbitos por meio de estratégias de vigilância em saúde, visando identificar seus fatores determinantes e subsidiar a adoção de medidas que possam prevenir a ocorrência de óbitos evitáveis (BRASIL, 2009). Uma vez que essa temática tem sido historicamente pouco explorada, apesar de sua importância em termos de saúde pública, a realização de estudos referentes à mortalidade fetal em seus diferentes enfoques torna-se fundamental.

\section{CONCLUSÃO}

Os resultados evidenciaram que os óbitos fetais ocorreram, em sua maioria, em gestantes com idade entre 20 e 29 anos, 8 a 11 anos de estudo e gestação única. Houve predominância de óbitos em fetos do sexo masculino, idade gestacional entre 37 e 41 semanas e baixo peso ao nascer ( 1500 a 2499 gramas). Observouse maior frequência em partos vaginais, no anteparto e ocorridos em hospitais. As principais causas foram Hipóxia intrauterina, Complicações da placenta, cordão umbilical e membranas e outras afecções originadas no período perinatal. Como contribuição, o presente estudo reforça a importância de se conhecer o perfil epidemiológico e os fatores que contribuem para o óbito fetal, a fim de que sejam estabelecidas políticas públicas de saúde que subsidiem a formulação e a implementação de ações que visem à sua prevenção e redução. O estudo apresentou como limitações o número reduzido de estudos recentes sobre essa temática e a qualidade da base de dados empregada (SIM), na qual a variável "causa de morte" apresentou uma quantidade considerável de "mortes fetais de causa não especificada", o que gera subinformação a respeito da verdadeira causa do óbito fetal.

\section{REFERÊNCIAS}

1. ASSIS HM, et al. Óbitos fetais sob o prisma da evitabilidade: análise preliminar de um estudo para o município de Belo Horizonte. Cadernos Saúde Coletiva, 2014; 22(3): 314-7.

2. BARBEIRO FMS, et al. Óbitos fetais no Brasil: revisão sistemática. Revista de Saúde Pública, 2015; 49(22): 1-13.

3. BARROS PS, et al. Mortalidade fetal e os desafios para a atenção à saúde da mulher no Brasil. Revista de Saúde Pública, 2019; 53(12).

4. BRASIL. Ministério da Saúde. Portaria $n^{\circ} 72$, de 11 de janeiro de 2010. Estabelece que a vigilância do óbito infantil e fetal é obrigatória nos serviços de saúde (públicos e privados) que integram o Sistema Único de Saúde (SUS). Disponível em: http://bvsms.saude.gov.br/bvs/saudelegis/gm/2010/prt0072_11_01_2010.html. Acesso em: 8 jan. 2020.

5. BRASIL. Ministério da Saúde. Secretaria de Atenção à Saúde. Departamento de Ações Programáticas Estratégicas. Gestação de alto risco: manual técnico. 5. ed. Brasília: Editora do Ministério da Saúde, 2012. 302 p.

6. BRASIL. Ministério da Saúde. Secretaria de Vigilância em Saúde. Secretaria de Atenção à Saúde. Manual de vigilância do óbito infantil e fetal e do Comitê de Prevenção do Óbito Infantil e Fetal. 2. ed. Brasília: Ministério da Saúde, 2009. $96 \mathrm{p}$.

7. BRITO MAMM, et al. Perfil obstétrico dos óbitos perinatais em uma capital do Nordeste Brasileiro. Revista Brasileira de Saúde Materno Infantil, 2019; 19(1): 259-267.

8. CHIAVEGATTO-FILHO ADP, LAURENTI R. O sexo masculino vulnerável: razão de masculinidade entre os óbitos fetais brasileiros. Caderno de Saúde Pública, 2012; 28(4): 720-728.

9. DATASUS. Departamento de Informática do Sistema Único de Saúde (SUS). Disponível em: http://www2.datasus.gov.br/DATASUS/index.php?area=02. Acesso em: 6 jan. 2020. 
10. FILHA FSSC, et al. Óbitos fetais e neonatais: análise do perfil sociodemográfico e clínico obstétrico de genitoras. Revista Científica de Enfermagem, 2019; 9(28): 92-102.

11. GIRALDI LM, et al. Óbito fetal: fatores obstétricos, placentários e necroscópicos fetais. Jornal Brasileiro de Patologia e Medicina Laboratorial, 2019; 55(1): 98-113.

12. HOLANDA AAS. Caracterização da mortalidade fetal em Pernambuco, de 2000 a 2011: causas e fatores associados. Monografia (Residência Multiprofissional em Saúde Coletiva), Centro de Pesquisas Aggeu Magalhães, Fundação Oswaldo Cruz. Recife: A autora, 2013; 61 p.

13. LIMA KJ, et al. Análise da situação em saúde: a mortalidade fetal na 10ª Região de Saúde do Ceará. Revista Brasileira em Promoção da Saúde, 2017; 30(1): 30-37.

14. MAZOTTI BR, et al. Fatores epidemiológicos correlacionados ao risco para morte fetal: revisão integrativa da literatura. Revista Arquivos de Ciências da Saúde, 2016; 23(2): 9-15.

15. MENEZZI AMED, et al. Vigilância do óbito fetal: estudo das principais causas. O Mundo da Saúde, 2016; 40(2): 208212.

16. OLIVEIRA EFV, et al. Gravidez na adolescência e outros fatores de risco para mortalidade fetal e infantil no Município do Rio de Janeiro, Brasil. Caderno de Saúde Pública, 2010; 26(3): 567-578.

17. Organização Mundial da Saúde (OMS). Classificação estatística internacional de doenças e problemas relacionados à saúde CID-10. Tradução centro colaborador da OMS para a família de Classificação Internacional em Português. $10^{\mathrm{a}}$ revisão. 8 ${ }^{\mathrm{a}}$ ed. São Paulo: Editora da USP, 2008.

18. SCHRADER G. Perfil epidemiológico e evitabilidade dos óbitos fetais de mulheres residentes em um município da região sul do Brasil. Dissertação (Mestrado em Ciências da Saúde) - Universidade do Sul de Santa Catarina, Palhoça, 2017; 120 p.

19. SILVA MMB, et al. Fatores associados ao óbito fetal em um hospital de referência em gestação de alto risco. Revista UNILUS Ensino e Pesquisa, 2017; 14(36): 68-80.

20. SILVA RMM, et al. Análise da incidência de óbitos fetais entre municípios da nona regional de saúde do Paraná. Semina: Ciências Biológicas e da Saúde, 2016; 37(1): 33-42.

21. SILVA VMC, et al. Fatores associados ao óbito fetal na gestação de alto risco: Assistência de enfermagem no prénatal. Revista Eletrônica Acervo Saúde, 2019; 37(37): 1-12. 